\title{
Advances in the management of multiple myeloma
}

\author{
James R Berenson, MD, ${ }^{\text {abc }}$ Tanya M Spektor, $\mathrm{PhD},{ }^{\mathrm{c}}$ and James Wang, $\mathrm{MD}^{\mathrm{ab}}$
}

${ }^{a}$ Institute for Myeloma and Bone Cancer Research; ${ }^{\mathrm{J} J a m e s} \mathrm{R}$ Berenson, MD, Inc; and ${ }^{\mathrm{C}}$ Oncotherapeutics, West Hollywood, California

How will you treat your next new patient with myeloma? What if the patient is or is not a transplant candidate? And what if he or she is progressing or relapsing and has bad cytogenetics?

Who would have thought 10 or more years ago that we would have so many new and effective therapies for multiple myeloma in the first, second, and beyond settings? Fast forward to now and the emerging science in understanding and treating myeloma has yielded new classes of drugs and new medications in each of the drug classes.

Here, Dr James Berenson and Dr Leslie Busby take us through this exciting and ever-expanding landscape of the new multiple myeloma therapies. Dr Berenson begins by defining what is normal, or MGUS (monoclonal gammopathy of undetermined significance), or smoldering myeloma, or myeloma. He follows with a discussion of each of the old and now new classes of medication and their mechanisms of action. Dr Busby looks at myeloma from the purely therapeutic angle and helps us make sense not only of the new drugs and their mechanisms of action, but how one might translate the available data for use in our daily practice and how these drugs might be sequenced as the patient moves along the myeloma trajectory.

So we thank Dr Berenson and $\operatorname{Dr}$ Busby (p. 235) for exploring and reviewing for us the immune-modulatory drugs, the proteasome inhibitors, the recent addition of the HDAC (histone deacetylase) inhibitors, and 2 exciting new antibodies, daratumumab against CD38 and elotuzumab against SLAMF7, which were approved for use in the United States last year. Auto- and allo-transplant approaches in first remission or in relapse/refractory disease remain another mainstay of therapy, although there remains controversy around the timing of auto-transplant and perhaps the findings of a large ongoing trial in the United States will provide guidance in the future. Myeloma has gone from being fatal disease within in a few years of diagnosis to being a chronic, more manageable condition now, and perhaps a cure for many patients is not too far off.

$$
\text { - David H Henry, MD, FACP }
$$

$\mathrm{M}$ ultiple myeloma $(\mathrm{MM})$ is a bone marrow-based malignancy of plasma cells that is diagnosed in over 30,000 patients annually in the United States. Despite the many recent advances in the treatment of MM, it remains an incurable disease. Thus, the need for the development of new effective therapies remains critical for these patients.

\section{Smoldering MM}

In general, it has not been shown that patients with smoldering MM (SMM) benefit from early treatment, but recent studies have identified a subset of patients who are at high-risk and may require therapy more quickly. Recent guidelines from the International Myeloma Working Group recommend immediate treatment of this subgroup of SMM. ${ }^{1}$ However, although findings in a Spanish study suggested that early treatment of high-risk SMM patients with the immunomodulatory agent (IMiD) lenalidomide and dexamethasone improves overall survival $(\mathrm{OS}),{ }^{2}$ the design of that study limits its clinical applicability, and no other randomized trials have been completed to show the advantage of early therapy for these patients.

\section{Specific drugs}

The development of novel agents such as proteasome inhibitors (PIs), IMiDs, histone deacetylase inhibitors (HDACIs), and monoclonal antibodies (mAbs) in recent years has vastly changed the approach to the treatment of MM patients.

PIs that are cytotoxic to MM cells, such as bortezomib, have become a foundation for MM treatment over the past decade. However, patients develop drug resistance to bortezomib by acquiring gene mutations and through other mechanisms. In recent years, newer forms of PIs such as carfilzomib and the oral formulations ixazomib and oprozomib have been and are currently being developed. ${ }^{3}$ Preclinical studies have shown that resistance to one PI can be overcome with treatment with another PI. ${ }^{4}$ That has recently

Correspondence: James R Berenson, MD; jberenson@imbcr.org. Disclosures: Dr Berenson is a consultant for and receives honoraria and research funding from Takeda, Amgen, Jansen, Celgene, and Bristol-Meyers-Squibb. Drs Spektor and Wang report no disclosures or conflicts of interest. JCSO 2016;14:232-238. (C2016 Frontline Medical Communications. doi: 10.12788/jcso.0270. 
been confirmed clinically in that replacement of bortezomib with carfilzomib has shown the ability to overcome resistance to bortezomib for most MM patients who receive a variety of bortezomib-containing combination treatments. ${ }^{5}$ A recent clinical trial has also shown improved progressionfree survival (PFS) when relapsed/refractory MM (RRMM) patients are treated with carfilzomib and the steroid dexamethasone, compared with bortezomib and dexamethasone. ${ }^{6}$ Ixazomib, an oral PI, was recently approved by the US Food and Drug Administration for the treatment of previously treated MM patients. ${ }^{78}$ Another novel PI, oprozomib, remains in clinical development. ${ }^{9}$

The IMiD lenalidomide has been approved not only for treating RRMM patients, but for use in the front-line setting as well. ${ }^{10}$ The drug has been combined with many other active anti-MM agents, which has enhanced its efficacy. ${ }^{11,12}$ Single-arm studies have shown high response rates when lenalidomide is combined with steroids and bortezomib. ${ }^{11}$ In addition, 2 recently reported randomized trials have shown an improved PFS when lenalidomide is combined with dexamethasone and either the PI carfilzomib or ixazomib, compared with the doublet of lenalidomide and dexamethasone without 1 of the newer agents. ${ }^{8,13}$ Another $\mathrm{IMiD}$, pomalidomide, has shown clinical efficacy among patients who are resistant to lenalidomide ${ }^{14}$ and is now being similarly combined with many other agents. ${ }^{15}$ Recent studies have shown high response rates when pomalidomide is combined with carfilzomib and dexamethasone in MM patients who are refractory to lenalidomide. ${ }^{16}$

In preclinical studies, the epigenetic modifying HDACIs have shown both single-agent activity and enhance the anti-MM effects of many other anti-MM agents including steroids, chemotherapeutic agents, PIs, and IMiDs. ${ }^{17,18}$ Panobinostat combined with bortezomib and dexamethasone has been approved for treating RRMM patients based on an improvement in PFS from the results of a large randomized study. ${ }^{19}$ However, the drug has significant side effects, especially gastrointestinal and constitutional symptoms, which have limited its use in the clinic.

Notably, a recent retrospective analysis of nearly 300 patients treated in our clinic showed that the response rates of MM patients who are treated with lenalidomide, bortezomib, or carfilzomib-containing regimens was similar across these 3 drugs in both the front-line and salvage settings. ${ }^{20}$ Notably, the findings also showed clinical benefit (minimal response or more) in about half of the patients when the same 3 drugs are used in a second, third, or even fourth combination treatment. In addition, substantial clinical activity was observed even among patients who were refractory to the drug in a previous combination treatment. High response rates were also observed among patients who had failed treatment with either carfilzomib or bortezomib and were then treated subsequently with brtezomib or carfilzomib, respectively.

\section{Maintenance therapy}

There have also been many advances in the use of maintenance therapy for MM patients. ${ }^{21}$ Findings in earlier maintenance trials had shown an improvement in OS from continuing steroids such as single-agent oral prednisone at therapeutic doses for MM patients who had responded to initial therapy with steroids and chemotherapy. ${ }^{22}$ Recent study findings have suggested the benefit of other drugs as maintenance therapy for patients who completed their initial therapy. ${ }^{21,23-25}$ Specifically, an improvement in OS with ongoing lenalidomide alone or with dexamethasone has been demonstrated in some but not all studies. ${ }^{23-25}$ Unfortunately, studies with the PIs have not defined their role as maintenance drugs, ${ }^{21}$ but the recent availability of the oral PI ixazomib will make the performance of these studies easier to undertake ${ }^{7}$.

\section{Immune-based treatments}

Recently, 2 mAbs, daratumumab and elotuzumab, have become available for treating RRMM patients. ${ }^{26-29}$ Daratumumab, which targets CD38, has shown significant clinical efficacy as a single agent but nearly half of the patients who are treated with it have experienced infusion reactions leading to prolonged infusion times or discontinuation of the drug. ${ }^{26}$ Elotuzumab, which targets signaling lymphocytic activation molecule family member 7 (SLAMF7), is present on both plasma cells and natural killer (NK) cells. ${ }^{30}$ This $\mathrm{mAb}$ is both directly cytotoxic to MM cells while activating the NK cell population, resulting in significant anti-MM effects. ${ }^{30}$ Although the drug lacks efficacy as a single agent, it improves PFS when combined with lenalidomide and dexamethasone with very few side effects. ${ }^{28}$ Many ongoing studies are evaluating these 2 mAbs with other anti-MM agents in the RRMM setting with promising early results. ${ }^{31,32}$ Other immune-based therapies that are currently in development include targeted antibody conjugates and chimeric antigen receptor (CAR)-modified T-cell-based treatments that have been used to effectively treat other B-cell malignancies. ${ }^{33,34}$ Despite the success of CAR T-cell treatment for some patients with CD19+ B-cell lymphoma, it has been extensively evaluated preclinically only in MM. A small number of MM patients have received this cellular therapy with some suggestion of clinical benefit but, in some cases, substantial toxicity including treatment-related deaths have occurred. ${ }^{34,35}$ However, an improvement in the cytotoxic potential of CAR T-cell therapies may lead to potentially better on target-specific therapy improving clinical outcomes for the treatment of MM patients.

\section{Medical problems and common side effects with $M M$ therapies}

Disease management includes not only the treatment of the disease but also other medical conditions. For instance, MM 
patients with anemia often have iron or B12 deficiency unrelated to the disease. ${ }^{36}$ Drugs commonly used to treat MM such as bortezomib and lenalidomide are also responsible for the occurrence of side effects such as peripheral neuropathy. Subcutaneous instead of intravenous administration, lower doses, and changes in schedule have been shown to reduce both the incidence and severity of PN from bortezomib treatment. ${ }^{37-40}$ In addition, the severity of peripheral neuropathy among patients treated with bortezomib or thalidomide is exacerbated by a concomitantly low serum vitamin D level. ${ }^{41}$ Lenalidomide has been associated with an increased risk of developing second primary malignances, ${ }^{42}$ although not all studies have shown this effect. ${ }^{43}$

\section{Summary}

Outcomes for MM patients are improving as a result of the recently approved new agents including mAbs. The place of these new drugs for the treatment of these patients as well as optimizing their use in combination with other effective anti-MM agents is the subject of many current clinical trials. Unfortunately, little remains known about the optimal sequencing or length of treatments for these patients; as more drugs become available, this will become even more difficult to sort out. Individualizing treatment for MM patients is also a goal for this disease so that patients can receive therapies based on the characteristics of their disease, immune system, and overall health, which should improve these patients' length and quality of life. Most importantly, these new treatments and learning that drugs can be re-used effectively and safely in new combinations have allowed patients to have an ever increasing number of options that have resulted in dramatic improvements in the quality and length of lives of our patients with MM.

\section{References}

1. Rajkumar SV, Dimopoulos MA, Palumbo A, et al. International Myeloma Working Group updated criteria for the diagnosis of multiple myeloma. Lancet Oncol. 2014;15:e538-e548.

2. Mateos MV, Hernandez MT, Giraldo P, et al. Lenalidomide plus dexamethasone for high-risk smoldering multiple myeloma. N Engl J Med. 2013;369:438-447.

3. Teicher BA, Tomaszewski JE. Proteasome inhibitors. Biochemical Pharmacol. 2015;96:1-9.

4. Sanchez E, Li M, Steinberg JA, et al. The proteasome inhibitor CEP18770 enhances the anti-myeloma activity of bortezomib and melphalan. Br J Haematol. 2010;148:569-581.

5. Berenson JR, Hilger JD, Yellin O, et al. Replacement of bortezomib with carfilzomib for multiple myeloma patients progressing from bortezomib combination therapy. Leukemia. 2014;28:1529-1536.

6. Dimopoulos MA, Moreau P, Palumbo A, et al. Carfilzomib and dexamethasone versus bortezomib and dexamethasone for patients with relapsed or refractory multiple myeloma (ENDEAVOR): a randomized, phase 3, open-label multicentre study. Lancet Oncol. 2016;17:27-38.

7. Offidani M, Corvatta L, Gentili S, Maracci L, Leoni P. Oral ixazomib maintenance therapy in multiple myeloma. Expert Review of Anticancer Therapy. 2016;1:21-32.

8. Moreau P, Masszi T, Grzasko N, et al. Ixazomib, an investigational oral proteasome inhibitor (PI), in combination with lenalidomide and dexamethasone (IRd), significantly extends progression-free survival (PFS) for patients (Pts) with relapsed and/or refractory multiple myeloma (RRMM): The Phase 3 Tourmaline-MM1 Study (NCT01564537) [ASH abstract 717]. Blood. 2015;126.

9. Kubiczkova L, Pour L, Sediarikova L, Hajek R, Sevickova S. Proteasome inhibitors - molecular basis and current perspectives in multiple myeloma. J Cell Mol Med. 2014;18:947-961.

10. Palumbo A, Hajek R, Delforge M, et al. MM-015 Investigators. Continuous lenalidomide treatment for newly diagnosed multiple myeloma. N Eng1 J Med. 2012;366:1759-1769.

11. Richardson PG, Weller E, Lonial S, et al. Lenalidomide, bortezomib, and dexamethasone combination therapy in patients with newly diagnosed multiple myeloma. Blood. 2010;116:679-686.

12. Berenson JR, Yellin O, Kazamel T, et al. A phase 2 study of pegylated liposomal doxorubicin, bortezomib, dexamethasone and lenalidomide for patients with relapsed/refractory multiple myeloma. Leukemia. 2012;26:1675-1680

13. Stewart AK, Rajkumar SV, Dimopoulos MA, et al. Carfilzomib, lenalidomide, and dexamethasone for relapsed multiple myeloma. $\mathrm{N}$ Engl J Med. 2015;372:142-152.

14. San Miguel JF, Weisel KC, Song KW. Impact of prior treatment and

depth of response on survival in MM-003, a randomized phase 3 study comparing pomalidomide plus low-dose dexamethasone versus high-dose dexamethasone in relapse/refractory multiple myeloma. Haematologica. 2015;100:1334-1339.

15. Berenson J, Stampleman L, Bessudo A, et al. A phase 1/2 trial of pomalidomide, dexamethasone and pegylated liposomal doxorubicin for patients with relapsed/refractory multiple myeloma (RRMM) [ASH abstract 5383]. Blood. 2015;126.

16. Shah JJ, Stadtmauer EA, Abonour R, et al. Carfilzomib, pomalidomide, and dexamethasone for relapsed or refractory myeloma. Blood. 2015; 126: 2284-2290.

17. Sanchez E, Shen J, Steinberg J, et al. The histone deacetylase inhibitor LBH 589 enhances the anti-myeloma effects of chemotherapy in vitro and in vivo. Leuk Res. 2011;35:373-379.

18. Hideshima T, Cottini F, Ohguchi H, et al. Rational combination treatment with histone deacetylase inhibitors and immunomodulatory drugs in multiple myeloma. Blood Cancer J. 2015;5:e312.

19. San-Miguel JF, Hungria VT, Yoon SS, et al. Panobinostat plus bortezomib and dexamethasone versus placebo plus bortezomib and dexamethasone in patients with relapsed or relapsed and refractory multiple myeloma: a multicenter, randomized, double-blind phase 3 trial. Lancet Oncol. 2014;15:1195-1206.

20. Berenson A, Vardanyan S, Ghermezi M, et al. Lack of significance of ISS staging and depth of response in predicting overall survival of multiple myeloma patients [ASH abstract 5771]. Blood. 2014;124.

21. Shank BR, Brown VT, Schwartz RN. Multiple myeloma maintenance therapy: a review of the pharmacologic treatment. J Oncol Pharm Pract. 2015;21:36-51.

22. Berenson JR, Crowley JJ, Grogan TM, et al. Maintenance therapy with alternate-day prednisone improves survival in multiple myeloma patients. Blood. 2002;99:3163-3168.

23. Palumbo A, Adam Z, Kropoff M, et al. A phase 3 study evaluating the efficacy and safety of lenalidomide combined with melphalan and prednisone followed by continuous lenalidomide maintenance (MPR-R) in patients $\geq 65$ years with newly diagnosed multiple myeloma (NDMM): updated results for patients aged 65-75 years enrolled in MM-015 [ASH abstract 475]. Blood. 2011;118.

24. Attal M, Lauwers-Cances V, Marit G. Lenalidomide maintenance after stem-cell transplantation for multiple myeloma. N Engl J Med 2012;366:1782-1791.

25. McCarthy PL, Owzar K, Hofmeister CC, et al. Lenalidomide after stem-cell transplantation for multiple myeloma. N Engl J Med. 2012;366:1770-1781.

26. Lokhorst HM, Plesner T, Laubach JP, et al. Targeting CD38 with daratumumab monotherapy in multiple myeloma. N Engl J Med. 
2015;373:1207-1219.

27. Chari A, Lonial S, Suvannasankha A, et al. Open-label, multicenter, phase $1 b$ study of daratumumab in combination with pomalidomide and dexamethasone in patients with at least 2 lines of prior therapy and relapsed or relapsed and refractory multiple myeloma $[\mathrm{ASH}$ abstract 508]. Blood. 2015;126.

28. Lonial S, Dimopoulos M, Palumbo A, et al. Elotuzumab therapy for relapsed or refractory multiple myeloma. $\mathrm{N}$ Engl J Med. 2015;373:621-631.

29. Berenson J, Swift R, Hobson S, Rifkin R. Elotuzumab administered over approximately 60 minutes in combination with lenalidomide and dexamethasone in patients with multiple myeloma: a phase 2 safety study [Abstract in press: HOPA 2016].

30. Hsi ED, Steinle R, Balasa B, et al. CS1, a potential new therapeutic antibody target for the treatment of multiple myeloma. Clin Cancer Res. 2008;14:2775-2784.

31. Plesner R, Arkenau H-T, Gimsing P, et al. Daratumumab in combination with lenalidomide and dexamethasone in patients with relapsed or relapsed and refractory multiple myeloma: updated results of a phase $1 / 2$ study (GEN503) [ASH abstract 507]. Blood. $2015 ; 126$.

32. Palumbo A, Offidani M, Pegourrie B, et al. Elotuzumab plus bortezomib and dexamethasone versus bortezomib and dexamethasone in patients with relapsed/refractory multiple myeloma: 2-year followup [ASH abstract 510]. Blood. 2015;126.

33. Munshi NC, Anderson KC. New strategies in the treatment of multiple myeloma. Clin Cancer Res. 2013;19:3337.

34. Atanackovic D, Radhakrishnan SV, Bhardwaj N, Luetkens T. Chimeric antigen receptor (CAR) therapy for multiple myeloma. $\mathrm{Br}$ J Haematol. 2016;172:685-698.

35. Linette GP, Stadtmauer EA, Maus MV, et al. Cardiovascular toxicity and titin cross-reactivity of affinity-enhanced T-cells in myeloma and melanoma. Blood. 2013;122:863-871.

36. Birgegard G. Managing anemia in lymphoma and multiple myeloma. Therapeutics and clinical risk management. 2008;4:527-539.

37. Moreau P, Pylypenko H, Grosicki S, et al. Subcutaneous versus intravenous administration of bortezomib in patients with relapsed multiple myeloma: a randomized phase 3, non-inferiority study. Lancet Oncol. 2011;12:431-440.

38. Jagannath S, Barlogie B, Berenson J, et al. A phase 2 study of two doses of bortezomib in relapsed or refractory myeloma. Br J Haematol. 2004;127:165-172.

39. Berenson JR, Yang HH, Sadler K, et al. Phase I/II trial assessing bortezomib and melphalan combination therapy for the treatment of patients with relapsed or refractory multiple myeloma. J Clin Oncol. 2006;24:937-944.

40. Berenson JR, Yellin O, Woytowitz D, et al. Bortezomib, ascorbic acid and melphalan (BAM) therapy for patients with newly diagnosed multiple myeloma: an effective and well-tolerated frontline regimen. Eur J Haematol. 2009; 82:433-439.

41. Wang J, Udd KA, Vidisheva A, et al. Low serum vitamin D occurs commonly among multiple myeloma patients treated with bortezo$\mathrm{mib}$ and/or thalidomide and is associated with severe neuropathy. Support Care Cancer. 2016. doi: 10.1007/s00520-016-3126-1.

42. Palumbo A, Bringhen S, Kumar SK, Lupparelli G, Usmani S. Second primary malignancies with lenalidomide therapy for newly diagnosed myeloma: a meta-analysis of individual patient data. Lancet Oncol. 2014;15:333-342.

43. Wang Y, Yang F, Shen Y, et al. Maintenance therapy with immunomodulatory drugs in multiple myeloma: a meta-analysis and systematic review. J Natl Cancer Inst. 2015; 108. doi: 10.1093/jnci/djv342.

\title{
How I treat multiple myeloma
}

\author{
Leslie T Busby, MD
}

Rocky Mountain Cancer Center, Boulder, Colorado

$\mathrm{I}$ love treating patients with multiple myeloma because it usually means the start of a long relationship. There are several highly active agents available to treat the disease and they are often safely combined to yield even greater responses and benefits for the patient. Unfortunately, there is an almost endless number of regimens and their corresponding acronyms. In addition, the number of quality, large-volume phase 3 studies on treating multiple myeloma are limited, making it difficult to know which of the dozens of available therapies is best. Many of the treatment guidelines, such as those from the National Comprehensive Cancer Network and mSMART, rely on expert opinions to help make sense of it all.

There are also 2 somewhat divergent opinions on how best to treat myeloma. One approach is to treat it aggressively with multiple chemotherapy agents, high-dose chemotherapy followed by stemcell rescue, and prolonged maintenance also using multiple agents. The other is to treat it as an incurable, chronic disease. In latter approach one works on getting the disease under control with a combination regimen and then keeping it under control, often using a single agent to minimize toxicity. Unfortunately, we don't know which method is best. I favor and treat patients using the latter approach because my patients tend to be older and have more comorbidities than those reported in research studies. Although I have had only a small number of patients who have gone through the more intense option, I have yet to see any cures.

When I have a newly diagnosed patient, I first determine if they even need therapy. Most patients

Correspondence: Leslie T Busby, MD; Leslie.Busby@USOncology.com. Disclosures: The author reports no disclosures or conflicts of interest. 


\section{Feature}

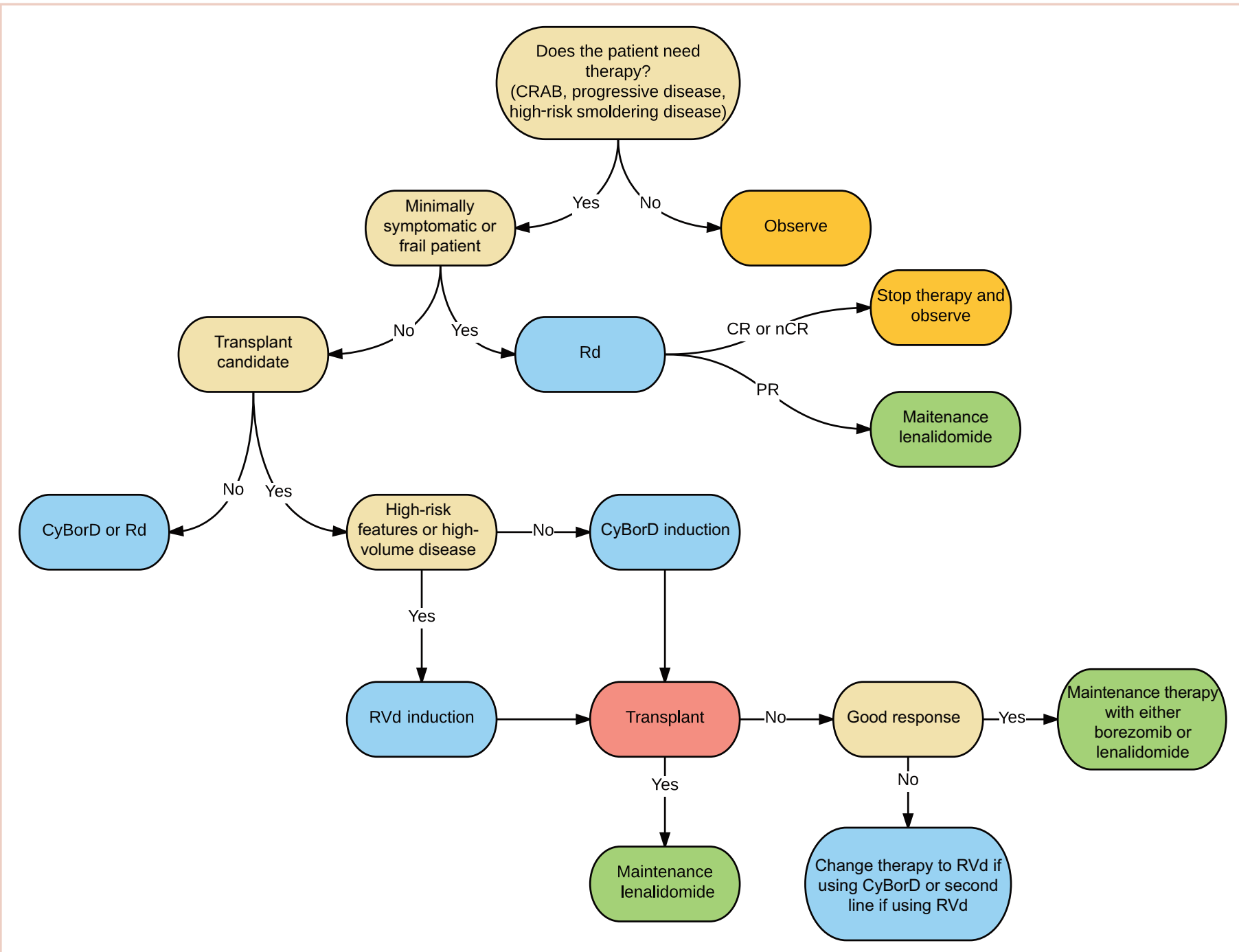

FIGURE First-line therapy: multiple myeloma algorithm

$\mathrm{CR}$, complete response; $\mathrm{CRAB}$, Calcium (elevated)+Renal failure+Anemia+Bone lesions; CyBorD, cyclophosphamide, bortezomib, and dexamethasone; $\mathrm{nCR}$, near-complete response; PR, pregression; Rd, dexamethasone; RVd, lenalidomide, bortezomib, and dexamethasone

with smoldering myeloma can be monitored. A Spanish study that compared lenalidomide and dexamethasone with best supportive care showed a survival advantage. ${ }^{1}$ However, because lenalidomide was not approved for firstline myeloma in Spain, the patients in the best supportive care group never received the drug when they progressed. Therefore, the study only proved that using lenalidomide in myeloma prolongs survival. I will use lenalidomide and weekly dexamethasone (Rd) in older or frail patients, especially those with relatively minimal symptoms. ${ }^{2}$ If they have a response, I will drop the dexamethasone after a few months and continue lenalidomide. I will also stop both drugs entirely if I get a complete response, which helps minimize both physical and financial toxicity.

The next assessment I make at presentation is how ill the patient is. As above, an elderly woman with isolated anemia or minimal bone involvement may do very well with $\mathrm{Rd}$. For patients with significant symptoms related to myeloma - hypercalcemia, significant renal dysfunction or failure, anemia, and significant bone involvement, together referred to as CRAB - my go-to regimen is cyclophosphamide, bortezomib, and dexamethasone (CyBorD). ${ }^{3}$ It is well tolerated, has a high rate of response, has minimal cytopenias, and is generally safe to use in the presence of renal disease. I administer bortezomib $1.3-1.5 \mathrm{mg} / \mathrm{m}^{2}$ both subcutaneously and weekly as this has been shown to have equivalent activity with less toxicity, especially peripheral neuropathy, compared with the original biweekly intravenous administration ${ }^{4,5}$.

I will use lenalidomide, bortezomib, and dexamethasone (RVd) if I need a more rapid response, if the patient has high-risk features such as a $17 \mathrm{p}$ - abnormality, or I am not 
getting a satisfactory response to $\mathrm{CyB}$ orD. There are different variations of RVd but the one I use most is a modified version published by Rajkumar, ${ }^{6}$ which is a 21 -day cycle of bortezomib $1.3 \mathrm{mg} / \mathrm{m}^{2}$ on days 1,8 , and 15 ; lenalidomide $25 \mathrm{mg}$ on days 1-14; and dexamethasone $40 \mathrm{mg}$ on days 1 , 8 , and 15 . It has a high response rate but also comes with more toxicity especially in elderly patients and patients with renal disease.

High-dose chemotherapy followed by stem-cell rescue is still considered standard therapy in patients with a good performance status but this too is being challenged in research protocols. I discuss this option with my patients and encourage an evaluation by our local transplant physicians. I still recommend it in eligible patients, especially those who would be considered at high risk of early relapse. Recently, I have had patients question the benefit of transplant. I have encouraged them to at least collect and store stem cells if they have had a good response to induction therapy.

Although data is limited, there is emerging information that continuing therapy or maintenance after reaching a maximum response or plateau phase prolongs time to next therapy. ${ }^{7}$ If I start a patient on CyBorD and they are not going for transplant, I drop the cyclophosphamide and dexamethasone once they have reached a plateau. I continue the bortezomib but with fewer injections, such as 3 weeks on and 1 week off or every other week. I also sometimes drop the dose from $1.3 \mathrm{mg} / \mathrm{m}^{2}$ to $1 \mathrm{mg} / \mathrm{m}^{2}$, especially if they are showing signs of peripheral neuropathy. If the patient undergoes high-dose chemotherapy followed by stem-cell rescue, I typically recommend maintenance lenalidomide $10 \mathrm{mg}$ per day, but I discuss the potential risks involved including secondary hematologic malignancies and cytopenias. ${ }^{8,9}$

Second-line therapy and beyond has become much more complex and many of my choices are dependent on how the patient is progressing, what he or she was already taking, and what toxicities are being experienced. If the patient was on lenalidomide, then I generally have gone to $\mathrm{CyB}$ orD similar to the induction regimen. If the patient was on bortezomib alone and adding cyclophosphamide and dexamethasone as in CyBorD was ineffective, I would often try to add another drug to bortezomib such as liposomal doxorubicin. However, the data of mixing a proteasome inhibitor, such as carfilzomib or ixazomib, with an immunomodulator, such as lenalidomide or pomalidomide, is very compelling. Carfilzomib, lenalidomide, and dexamethasone (KRd) had a high response rate of $73 \%$ and progression-free survival of 26.3 months in patients who had 1-3 previous therapies and did not immediately progress on lenalidomide just prior to entering the study. ${ }^{10}$ This is a 28 -day regimen, with carfilzomib given at $27 \mathrm{mg} / \mathrm{m}^{2}$ on days $1,2,8,9,15$, and 16 with a starting dose of $20 \mathrm{mg} / \mathrm{m}^{2}$ for days 1 and 2 of the first cycle only. Lenalidomide was given at $25 \mathrm{mg}$ on days 1-21 and dexamethasone was 40 $\mathrm{mg}$ on days $1,8,15$, and 22 . In my experience, this regimen is very active but needs to be monitored closely because it also causes significant fatigue and low blood counts especially in the elderly. If I am going to use it in a patient who may be on the edge of frailty, I will lower the dose of lenalidomide to $10-15 \mathrm{mg}$ daily. We are starting to get some clarity, but it has taken several years since carfilzomib was approved to better understand the best dose, schedule, and combinations. Because there is activity in weekly dosing, ${ }^{11} \mathrm{I}$ have reduced it to weekly for patients who are not tolerating the standard biweekly and, if they are doing well, I try to increase the individual dose.

Panobinostat, a histone deacetylase inhibitor, along with bortezomib and dexamethasone was approved last year in patients who have received previous bortezomib and an immunomodulating therapy. ${ }^{12}$ The combination has $25 \%$ grade 3/4 risk of diarrhea, which has me and my patients reluctant to try it. Ixazomib, an oral proteasome inhibitor, and elotuzumab, a SLAMF7 monoclonal antibody, each given along with lenalidomide and dexamethasone were both approved last year in patients who have received 1-3 previous treatments. ${ }^{13,14}$ Both regimens were shown to be superior to lenolidamide and dexamethasone alone, however, there is no information as to whether either of those is better than RVd or KRd. In addition, there is limited data on single-agent ixazomib, and monotherapy with elotuzumab does not seem to be active.

Daratumumab has single-agent activity in patients who have progressed on both a proteasome inhibitor and lenolidamide. ${ }^{15}$ There are concerns regarding reactions leading to prolonged infusions, especially with the first dose, but most of these are mild. Because of the first-week reactions, a potentially long infusion time, and its relatively short shelf life, it is difficult to give it per the package insert in the outpatient setting. Working with many members in our group, we developed our own regimen, splitting the infusion over 2 days for the first week only. This is not in accordance with the package insert but thus far seems to be working well. This has also allowed us to keep our patients in the clinic rather than sending them to the hospital. The most common problem I have had with the drug has been primarily related to the significant amount of steroids recommended. Daratumumab can also bind to CD38 present on red blood cells and can interfere with serologic testing because of a false positive Coombs test. It also masks antibody detection to minor antigens in the patient's serum. If the patient requires a blood transfusion, your blood bank needs to be notified that the patient received daratumumab.

Myeloma is a complex family of diseases that cannot be treated by a simple "cookbook" approach. This is why I find treating this disease both challenging and stimulating. Although cures are rare, the newer and highly effective 
therapies are allowing more patients to live with their disease for years, some past a decade. They also give me hope that I will see more complete responses in my patients. None of these advancements would have been possi-

\section{References}

1. Mateos MV, Hernandez MT, Giraldo P, et al. Lenalidomide plus dexamethasone for high risk smoldering multiple myeloma. N Engl J Med. 2013;369:438-447.

2. Benboubker L, Dimopoulos MA, Dispenzieri A, et al. Lenalidomide and dexamethasone in transplant-ineligible patients with myeloma. N Engl J Med. 2014;371:906-917.

3. Reeder CB, Reece DE, Kukreti V, et al. Once- versus twice-weekly bortezomib induction therapy with $\mathrm{CyB}$ orD in newly diagnosed multiple myeloma. Blood. 2010;115:3416-3417.

4. Moreau P, Pylypenko H, Grosicki S, et al. Subcutaneous verses intravenous administration of bortezomib in patients with relapsed multiple myeloma: a randomized, phase 3 , non-inferiority study [Erratum in Lancet Oncol. 2011;12:522.]. Lancet Oncol. 2011;12:431-440.

5. Bringhen S, Larocca A, Rossi D, et al. Efficacy and safety of once-weekly bortezomib in multiple myeloma patients. Blood. 2010;116:4745-4753.

6. Rajkumar SV. Multiple myeloma: 2011 update on diagnosis, risk-stratification, and management [Erratum in Am J Hematol. 2014;89:669. Am J Hematol. 2011;8:57-65.

7. Palumbo A, Gay F, Cavallo F, et al. Continuous therapy versus fixed duration of therapy in patients with newly diagnosed multiple myeloma. J Clin Oncol. 2015;33:3459-3466.

8. McCarthy PL, Owzar K, Hofmeister CC, et al. Lenalidomide after stem-cell transplantation for multiple myeloma. N Engl J Med. 2012;366:1770-1781.

9. Palumbo A, Bringhen S, Kumar SK, et al. Second primary malig- ble without the participation of hundreds of patients on clinical trials, and I encourage you to help continue these advancements by enrolling your patients in clinical trials when they are available.

nancies with lenalidomide therapy for newly diagnosed myeloma: a meta-analysis of individual patient data. Lancet Oncol. 2014;15:333-342.

10. Stewart AK, Rajkumar SV, Dimopoulos MA, et al. Carfilzomib, lenalidomide, and dexamethasone for relapsed multiple myeloma. $\mathrm{N}$ Engl J Med. 2015;372:142-152.

11. Berenson J, Cartmell A, Lyons R, et al. Weekly carfilzomib with dexamethasone for patients with relapsed or refractory multiple myeloma: updated results from the phase $1 / 2$ study champion- 1 [ASH abstract 373]. https://ash.confex.com/ash/2015/webprogram/ Paper79628.html. Presented December 2015. Accessed May 4, 2016.

12. San-Miguel JF, Hungria VT, Yoon SS, et al. Panobinostat plus bortezomib and dexamethasone versus placebo plus bortezomib and dexamethasone in patients with relapsed or relapsed and refractory multiple myeloma: a multicentre, randomised, double-blind phase 3 trial [Erratum in Lancet Oncol. 2015;16:e6]. Lancet Oncol. 2014;15:1195.

13. Moreau P, Masszi T, Grzasko N, et al. Oral ixazomib, lenalidomide, and dexamethasone for multiple myeloma. N Engl J Med. 2016;274:1621-1634.

14. Lonial S, Dimopoulos M, Palumbo A, et al. Elotuzumab therapy for relapsed or refractory multiple myeloma. N Engl J Med. 2015;373:621-631.

15. Lokhorst HM, Plesner T, Laubach JP, et al. Targeting CD38 with daratumumab monotherapy in multiple myeloma. N Engl J Med. 2015;373:1207-1219. 